On page 363 in Vol. 16, No. 3 in the March issue, the authors' names were inadvertently run in, removing the spaces between the first and last names, with extraneous superscripts after them; an inadvertent error which the printer sincerely regrets. The correct version of the author line appears below.

\title{
Automated Nanospray Using Chip-Based Emitters for the Quantitative Analysis of Pharmaceutical Compounds
}

\author{
Leonard J. Corkery* and Henrianna Pang
}

Eli Lilly Canada Inc., Toronto, Ontario, Canada

\author{
Bradley B. Schneider and Thomas R. Covey
}

MDS SCIEX, Concord, Ontario, Canada

K. W. Michael Siu

Department of Chemistry and Center for Research in Mass Spectrometry, York University, Toronto, Ontario, Canada

An automated nanospray system based on chip technology (the NanoMate) was successfully interfaced to a modified Particle Discriminator Interface on a triple quadrupole mass spectrometer. A number of the interface parameters were optimized to improve the sampling efficiency for ions from the chip-based system. Analytical performance was assessed using a number of biochemicals as well as via a methodology for a pharmaceutical that passed validation as required by Good Laboratory Practices. Infusion analyses in flow rates $<1$ $\mu \mathrm{L} / \mathrm{min}$ provided advantages in terms of throughput and sample consumption when compared to other methodologies based on liquid chromatography. (J Am Soc Mass Spectrom 2005, 16, 363-369) (C) 2004 American Society for Mass Spectrometry 